\title{
Involved Nodal Radiotherapy vs. Involved Field Radiotherapy after Chemotherapy in the Treatment of Early Stage Hodgkin's Lymphoma*
}

\author{
Hamdy M. Zwam, Emad E. Habib", Mustafa E. AL-Daly \\ Clinical Oncology Department, Faculty of Medicine, Cairo University, Cairo, Egypt. \\ Email: "e.habib@kasralainy.edu.eg
}

Received December $8^{\text {th }}, 2012$; revised January $10^{\text {th }}, 2013$; accepted January $18^{\text {th }}, 2013$

\begin{abstract}
Aim of Work: This study is a prospective randomized trial aiming to investigate whether radiotherapy volume can be reduced without loss of efficacy from involved field radiotherapy (IFRT) to involved node radiotherapy (INRT) after four cycles of ABVD chemotherapy in the treatment of early stage Hodgkin's lymphoma. Patients and Methods: Between September 2009 and January 2012, all patients with newly diagnosed early-stage favorable and unfavorable Hodgkin's lymphoma attending to the Clinical Oncology department of Cairo University, faculty of medicine were enrolled into this study after a written consent was obtained from those cases enrolled. Patients were assigned to receive (ABVD) for four cycles followed by randomization for radiotherapy into two arms one arm of 30 Gy INRT $+/-6$ Gy to residual disease or another arm of $30 \mathrm{~Gy} \mathrm{IFRT}+/-6$ Gy to residual disease. Results: 35 patients were enrolled in this study: 16 patients in the INRT arm and 19 patients in the IFRT arm. The median observation time was 25 months. The overall survival for all eligible patients was $97 \%$ and freedom from treatment failure was $85.7 \%$. Survival rates at the end of the study revealed no differences between INRT and IFRT arms. Also, in terms of complete remission post radiotherapy (14 versus 15$)$, relapse (1 versus 4$)$, and death ( 0 versus 1$)$ respectively the outcome was similar in both arms. As regard acute and sub-acute toxicities no significant difference could be detected between both arms except that IFRT arm was associated with a higher incidence of radiation pneumonitis (4 versus 1 patient). Conclusion: Radiotherapy volume size reduction from IFRT to INRT after ABVD chemotherapy for four cycles produces similar results and less toxicity in patients with early-stage Hodgkin's lymphoma especially in patients with mediastinal disease.
\end{abstract}

Keywords: Hodgkin’s Lymphoma; Nodal Radiotherapy; Field Radiotherapy

\section{Introduction}

Hodgkin's lymphoma through the past 50 years has been at the center of the development of modern oncologic therapeutics, and investigation [1].

The incidence of Hodgkin's lymphoma shows marked heterogeneity with respect to age, gender, race, geographic area, social class and histological subtype [2].

Hodgkin's lymphoma is listed as a rare disease by the office of rare diseases (ORD) of the National Institutes of Health (NIH). Worldwide prevalence rates vary, with more than 5.5 per 100,000 in Yemen and Lebanon and less than 1 per 100,000 in China and Japan. At least some of this variation appears to relate to the degree of industrialization [1].

Notably, Hodgkin's lymphoma has 2 age-specific incidence peaks in developed countries: 15 - 34 years of

\footnotetext{
"The authors declare no conflict of interest.

\#Corresponding author.
}

age and older than 50 years of age, although these peaks are composed mainly of different subtypes with nodular sclerosis pathology being predominantly represented in the earlier age peak and the mixed cellularity disease being predominant in the later age peak [3].

Currently combination chemotherapy and radiation therapy are the standards of care for adults with limited-stage HL. Treatment usually consists of two to four cycles of ABVD chemotherapy followed by radiation therapy to the sites of initial disease involvement $[2,3]$.

While Radiation treatment remains a key component in combined modality therapy for patients with early stage HL. Its use has been questioned due to late complications, including cardiac and pulmonary toxicities, secondary malignancy etc. Radiation toxicity is dependent on the irradiated volume and radiation dose [4-6].

A recent review of relapses in patients treated with chemotherapy alone showed that most recurrences occurred in the initially involved lymph nodes [7]. With 
modern techniques, including better $\mathrm{CT}$ scan imaging, FDG-PET/CT scans and more accurate radiation technology, it is now possible to customize radiotherapy for each patient with accurate delivery of radiation to the initially involved volume while minimizing the radiation dose to normal tissues. The concept of involved field radiotherapy (IFRT) may now be replaced by the concept of involved-node radiotherapy (INRT), which only includes the initially involved lymph node(s). Better sparing of normal tissues is expected with the use of INRT compared with conventional IFRT provided that the initial tumor mass is not too large and involved lymph nodes are not too many [8].

Early response to PET has been identified as a powerful prognostic tool in Hodgkin's lymphoma. Two prospective trials published recently evaluated the prognostic role of an early interim scan (PET 2) in Hodgkin's lymphoma patients after two cycles of conventional ABVD chemotherapy. Consolidation radiotherapy was delivered only to patients with bulky disease at presentation or residual disease. The 2-year progression-free survival for patients with positive PET 2 scan results was only $12.8 \%$ when compared with $95 \%$ for patients with negative PET 2 scan results. The PET 2 was the single significant prognostic factor predicting treatment outcome on multivariate analysis, surpassing the prognostic value of the international prognostic score $[9,10]$ leading the authors to conclude that four cycles of ABVD followed by 30 Gy of IFRT is the optimal therapy for earlystage, unfavorable Hodgkin's lymphoma [10].

\section{Aim of Work}

To compare involved nodal radiotherapy technique treatment outcome versus involved field technique in early stage Hodgkin's lymphoma in terms of locoegional control, disease free survival and treatment morbidity.

\section{Patients and Methods}

\subsection{Eligibility Criteria}

- Histologically proven Hodgkin's lymphoma, with exclusion of the lymphocyte predominant subtype.

- Clinical stage I or II, both favorable and unfavorable prognostic subsets, with tumor bulk less or equal to $10 \mathrm{~cm}$.

- Previously untreated.

- Age: between 16 and 75 years.

- Good general condition (WHO performance status $0-1-2)$.

- Free of concurrent disease. Patients with impaired heart, lung, liver, or kidney function; previous malignant disease or HIV-positive status are not included in this study.

- Pregnant or lactating are excluded.

\subsection{Work Up}

Routine staging procedures included medical history; physical examination; computed tomography of the chest, abdomen, and pelvis; bone marrow biopsy (for unfavourable cases); $\mathrm{CBC}$; serum chemistry and echocardiography.

\subsection{Study Design}

This study is a prospective randomized trial comparing the outcome of consolidation radiotherapy techniques either by using INRT or IFRT in the treatment of (stage I \& II) supra-diaphragmatic Hodgkin's lymphoma.

IFRT was defined as radiation therapy fields that encompass the initially involved nodal regions only. This radiation therapy field is defined to cover the initially involved lymph nodes plus contiguous nodal groups. INRT is defined as radiation therapy to treat the initially involved lymph nodes only.

All eligible patients will be stratified according to the classic EORTC clinical prognostic factors into the unfavourable and favourable subsets as follows:

- Unfavourable subset includes patients with: Clinical stage II with 4 or more nodal areas or an age of 50 or more years or mediastinal lymphadenopathy more than a third of the chest width or an ESR $\geq 50$ (without B-symptoms) or ESR $\geq 30$ (with B-symptoms).

- Favorable subset: Includes all other patients with criteria not applicable to the unfavourable subset.

\subsection{Primary Endpoints}

- Progression-free survival (PFS).

- Loco-regional control.

\subsection{Secondary Endpoint}

- Event free survival (EFS).

- And late toxicity.

\subsection{Administered Chemotherapy}

\section{ABVD Regimen:}

\begin{tabular}{lll}
\hline Doxorubicin & $25 \mathrm{mg} / \mathrm{m}^{2}$ i.v. & day 1 and 15 \\
\hline Bleomycin & $10 \mathrm{mg} / \mathrm{m}^{2}$ i.v. & day 1 and 15 \\
Vinblastine & $6 \mathrm{mg} / \mathrm{m}^{2}$ i.v. & day 1 and 15 \\
Dacarbazine & $375 \mathrm{mg} / \mathrm{m}^{2}$ i.v. & day 1 and 15 \\
\hline
\end{tabular}

Treatment usually consists of four cycles of ABVD. Complete blood counts should be obtained before each intravenous drug administration, day 1 and day 15 . 
The treatment of the first cycle should be given at a $100 \%$ dosage. Unless a life-threatening toxicity including infection occurs before day 15, full doses of ABVD will also be given on day 15 of the first cycle. If a life-threatening toxicity has occurred, treatment should be postponed for a maximum of two weeks and treatment should be resumed after decrease of the toxicity to grade 0,1 or 2 with the day 15 schedule at full dose. When life-threatening toxicity persists or only decreases to grade 3 beyond two weeks, treatment is stopped and the patient will be excluded from the study because of intolerance to treatment.

\subsection{Radiotherapy}

IFRT Arm is implemented using the definitions and guidelines of Noordijk and Yahalom [11,12].

INRT Arm is carried out with the recommendations of EORTC-GELA radiotherapy group of $\mathrm{H} 10$ and GHSG HD 17 study with adjustment according to our facilities [13-15].

General guidelines for both arms:

- Patients must be examined by the radiation oncologist before chemotherapy.

- All patients must have pre- and post-chemotherapy cervical and thoracic CT scans (axillary lymph node areas must be clearly visible on thoracic CT scans).

- CT scans should be performed in the treatment position as well as the pre-chemotherapy PET-CT, which can help pinpoint previously undetected involved lymph nodes.

- CT simulation, 3D-conformal radiotherapy, and immobilization devices are strongly recommended for proper implementation of involved node radiotherapy.

- The remission status after chemo-therapy should be determined for each initially involved lymph node exclusively using CT scans. Complete remission (CR) is defined as the complete disappearance of clinically and/or radiologically detectable disease. A complete remission unconfirmed $(\mathrm{Cru})$ is defined as at least a $75 \%$ decrease in tumor size. A partial response (PR) is at least a $50 \%$ decrease in tumor size. Failure is less than a $50 \%$ decrease or any increase in tumor size.

- Radiotherapy should start within 3-4 weeks after the end of the last chemotherapy course. The prescribed dose is 30 Gy with a boost of 6 Gy to areas with residual disease.

- The Gross Tumour Volume (GTV) (In case of partial remission) represents the lymph node remnant(s) and should be contoured first in such condition.

- The Clinical Target Volume (CTV) is the initial volume of the lymph node(s) before chemotherapy. In other words, the CTV incorporates the initial location and the extent of the disease and takes into account the displacement of normal structures. In case of a $\mathrm{CRu}$ with a visible lymph node remnant, the lymph node remnant should be included.

- The Planning Target Volume (PTV) is the CTV with a margin to take into account organ movement and set-up variations. In most situations, a $1 \mathrm{~cm}$ safety margin is considered adequate.

The PTV must receive 30 Gy. For patients in partial remission initial PTV must receive 30 Gy and the boost to the PTV must receive an additional dose of $6 \mathrm{~Gy}$.

\subsection{Evaluation of Response and Follow-Up}

Patients are monitored during therapy by physical examination, chest X-ray and routine blood tests. Computed tomography scans are performed 2 weeks after the last chemotherapy cycle and are followed by radiotherapy. The final restaging will be conducted 8 weeks after the end of radiotherapy. Patients then will be observed at 3 -month intervals during the first year, every 4 months in the second year. Freedom from treatment failure is defined as the time from the start of radiotherapy to the first of one of the following: Progressive disease (defined as appearance of new lesions or B symptoms, or an increase in any lesion of $25 \%$ in the largest diameter under treatment or within 3 months after the end of treatment). Relapsing disease (defined as appearance of new lesions or as reappearance of initial lesions or B symptoms after a period of at least 3 months of complete remission).

Statistical Methods: Data management and analysis were performed using Statistical Package for Social Sciences (SPSS) version 17. Numerical data were summarized using means and standard deviations. Categorical data were summarized as percentages. Comparisons between the two groups with respect to numeric variables were done by Mann-Whitney test, a nonparametric test equivalent to the Student's t test. The chi-square test or Fisher's exact test was used to compare between the groups with respect to categorical data. Progression free survival time was estimated using the methods of Kaplan and Meier. Differences between survival curves were assessed for statistical significance with the log-rank test. All p-values are two-sided. p-values $<0.05$ were considered significant.

\section{Results}

This study is a prospective randomized trial comparing the outcome of consolidation radiotherapy techniques either using INRT or IFRT in the treatment of stage I \& II supra-diaphragmatic HL.

Starting from September 2009 till January 2012, 35 patients attending to Kasr Al-ainy Centre of Clinical 
Oncology, Cairo University, representing all stage I - II supra-diaphragmatic Hodgkin's lymphoma referred to our Centre during this period of time were included. Sixteen patients were included in the INRT arm and 19 patients in the IFRT arms.

\subsection{Patient's Characteristics}

The patient characteristics are demonstrated in Table 1.

Regarding international prognostic score (IPS) patient related factors applicable to early Hodgkin's lymphoma which include: age, ESR, LDH, total leucocytic count, hemoglobin, lymphocytic count and serum albumen were determined for both arms and was higher for the IFRT arm with a maximum score of 4 . On statistical comparison of both arms the p-value was 0.006 as demonstrated in Table 2.

Performance status at presentation was also determined for both groups with no significant difference between them $(\mathrm{p}=0.713)$.

\subsection{Treatment Outcome}

All patients underwent $\mathrm{CT}$ scan assessment of the sites of the initial disease before the third cycle and after ending the fourth cycle of chemotherapy and $4-6$ weeks after

Table 1. Patient characteristics for both arms.

\begin{tabular}{|c|c|c|c|c|c|}
\hline Factor & \multicolumn{2}{|c|}{ INRT Arm } & \multicolumn{2}{|c|}{ IFRT Arm } & p-value \\
\hline Age range & \multicolumn{2}{|c|}{$18-59$ years } & \multicolumn{2}{|c|}{$20-60$ years } & 07 \\
\hline Median age & \multicolumn{2}{|c|}{26 years } & \multicolumn{2}{|c|}{29.5 years } & 0.1 \\
\hline Males:Females & \multicolumn{2}{|c|}{$5: 11$} & \multicolumn{2}{|c|}{$13: 6$} & 0.028 \\
\hline Pathological subtype & $\#$ of cases & $\%$ & \# of cases & $\%$ & P-value \\
\hline Nodular sclerosis & 10 & $66.6 \%$ & 9 & $47.3 \%$ & \multirow{4}{*}{0.067} \\
\hline Mixed cellularity & 5 & $31.25 \%$ & 8 & $42.1 \%$ & \\
\hline Lymphocyte predominant & 1 & $6.25 \%$ & 2 & $10.5 \%$ & \\
\hline Lymphocyte depletion & 0 & $0 \%$ & 0 & $0 \%$ & \\
\hline Stage I & 5 & $31 \%$ & 11 & $58 \%$ & \multirow{2}{*}{0.8} \\
\hline Stage II & 11 & $69 \%$ & 8 & $42 \%$ & \\
\hline A (no B symptoms) & 10 & $62.5 \%$ & 11 & $58 \%$ & \multirow{2}{*}{0.11} \\
\hline B symptoms & 6 & $37.5 \%$ & 8 & $42 \%$ & \\
\hline Favourable cases & 5 & $31 \%$ & 9 & $47 \%$ & \multirow{2}{*}{0.33} \\
\hline Unfavourable cases & 11 & $69 \%$ & 10 & $53 \%$ & \\
\hline
\end{tabular}

Table 2. IPS characteristics for patients of both arms.

\begin{tabular}{|c|c|c|c|c|c|c|c|c|c|}
\hline & \multicolumn{8}{|c|}{ RTH Technique } & \multirow[b]{3}{*}{$\mathrm{p}$-value } \\
\hline & \multicolumn{4}{|c|}{ INRT } & \multicolumn{4}{|c|}{ IFRT } & \\
\hline & $\mathrm{N}$ & Median & Minimum & Maximum & $\mathrm{N}$ & Median & Minimum & Maximum & \\
\hline Age & 16 & 29.5 & 20 & 60 & 19 & 26 & 18 & 59 & 0.678 \\
\hline ESR1 & 12 & 24 & 2 & 95 & 16 & 21.5 & 3 & 83 & 0.780 \\
\hline ESR2 & 12 & 48.5 & 11 & 99 & 18 & 18 & 1 & 112 & 0.034 \\
\hline $\mathrm{LDH}$ & 15 & 227 & 11 & 642 & 19 & 241 & 17 & 822 & 0.488 \\
\hline TLC & 16 & 5.4 & 3.6 & 22.5 & 19 & 5.4 & 3.7 & 22.9 & 0.436 \\
\hline HB & 16 & 12.1 & 1.2 & 14.9 & 19 & 11.8 & 1.2 & 17.6 & 0.934 \\
\hline Lymph. Count & 16 & 2.1 & 0.6 & 3.9 & 18 & 2.4 & 1.1 & 8.5 & 0.055 \\
\hline Int. prog. score & 16 & 1 & 0 & 3 & 19 & 2 & 1 & 4 & 0.006 \\
\hline
\end{tabular}


radiotherapy and then during routine regular follow-up.

The response rate to chemotherapy is expressed in Table 3.

Radiation therapy dose was determined depending on response. Patients in CR \& CRu received $30 \mathrm{~Gy} / 15 \mathrm{~F} / 3$ weeks while patients with $\mathrm{PR}$ received an additional boost of $6 \mathrm{~Gy}$ to residual disease. This approach is applied for either group.

The response rate after ending radiotherapy is summarized in Table 4.

After a median follow-up of 25 months (ranging from 12 to 48 months) relapses in both arms were a total of five cases. One case in the INRT and 4 in the IFRT arm. Regarding relapsing cases all of them relapsed in the initially involved sites before starting treatment.

The 2 years progression free survival for the INRT group is $91.7 \%$ and for the IFRT group it is $83.6 \%$, with no statistically significant difference between both groups as represented in Figure 1.

\subsection{Toxicity}

Patients of both arms where assessed for toxicity according to the Common Terminology Criteria for Adverse

Table 3. Response after chemotherapy.

\begin{tabular}{ccccc}
\hline Number of Cycles. & Response & INRT & IFRT & p-value \\
\hline & & 12 & 14 & \\
After 2 cycles & & $75.0 \%$ & $73.7 \%$ & \\
& PR & 4 & 5 & \\
& & $25.0 \%$ & $26.3 \%$ & \\
& CR & 2 & 3 & 1.000 \\
After 4 cycles & & $12.5 \%$ & $15.8 \%$ & \\
& & 10 & 11 & \\
& & $62.5 \%$ & $57.9 \%$ & \\
& & 4 & 5 & \\
& & & & \\
& & & $25 \%$ & $26 \%$ \\
\hline
\end{tabular}

Table 4. Response after radiotherapy.

\begin{tabular}{cccc}
\hline Response & INRT & IFRT & p-value \\
\hline $\mathrm{CR}$ & 4 & 5 & \\
& $25.0 \%$ & $26.3 \%$ & \\
$\mathrm{CRu}$ & 10 & 10 & 0.666 \\
& $62.5 \%$ & $52.6 \%$ & \\
$\mathrm{PR}$ & 2 & 4 & \\
\hline
\end{tabular}

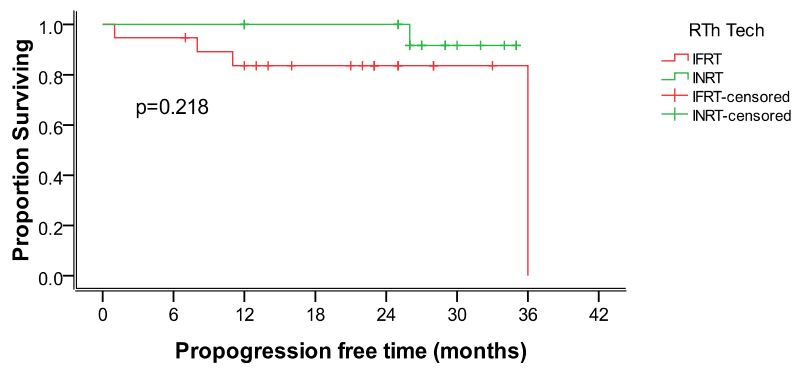

Figure 1. The 2 years progression free survival for both groups.

Events (CTCAE), version 3.0. The most frequently occurring toxicities included skin changes, dysphagia, mucositis, laryngeal toxicity, and pulmonary symptoms suggesting radiation pneumonitis. No statistically significant difference could be seen between both arms as regards skin $(p=0.115)$, dysphagia, $(p=0.728)$ or dysphonia $(p=0.830)$ while for radiation pneumonitis 5 cases were reported of a total 14 patients receiving mediastinal irradiation. Of these cases 1 patient was in the INRT arm and 4 patients were in the IFRT arm with a $p$ value equal to 0.58 .

None of the toxicities encountered in this trial were higher than grade II.

\subsection{Dose to Organs at Risk}

The reduction of PTV volume from IFRT to INRT resulted in reduction of the volume of included risk structures which is expressed in Table 5. As an example the dose to both lungs is reduced with a statistically significant reduction in V1, V5 and V20 on comparing both treatment arms as shown in Table 6.

Also, reduction of exposure for both the left and right breasts was found in V1 and D50\% with a statistically significant p-value as shown in Table 7.

Regarding, dose distribution to the heart there was no statistically significant difference between both groups. The thyroid gland exposure was reduced in the V1 and V5 in the INRT arm as shown in Table 8.

In general, to sum up matters median volumes of radiation exposure in the INRT group of patients was smaller than that in the IFRT group with the added benefit of reduction of overall radiation toxicity.

\section{Discussion}

A combined treatment modality is currently the state of art in the management of early Hodgkin's lymphoma. A treatment success of $80 \%$ to $90 \%$ was achieved using chemo-radiotherapy and was confirmed by many phase III trials as well as a meta-analysis $[3,16,17]$.

In spite of the recognized role of radiation therapy in 
Table 5. Comparison of the total PTV between both treatment arms.

\begin{tabular}{|c|c|c|c|c|c|c|c|c|c|}
\hline \multicolumn{10}{|c|}{ RTH Technique } \\
\hline & \multicolumn{4}{|c|}{ INRT } & \multicolumn{4}{|c|}{ IFRT } & \multirow[b]{2}{*}{$\mathrm{p}$-value } \\
\hline & $\mathrm{N}$ & Median & Minimum & Maximum & $\mathrm{N}$ & Median & Minimum & Maximum & \\
\hline PTV Total & 16 & 217 & 23.7 & 1127 & 19 & 683 & 3.8 & 2285 & 0.009 \\
\hline
\end{tabular}

Table 6. Comparison of different dosimetric parameters for both groups in case of mediastinal irradiation.

\begin{tabular}{|c|c|c|c|c|c|c|c|c|c|}
\hline \multicolumn{10}{|c|}{ Radiotherapy Technique } \\
\hline & \multicolumn{4}{|c|}{ INRT } & \multicolumn{5}{|c|}{ IFRT } \\
\hline & $\mathrm{N}$ & Median & Minimum & Maximum & $\mathrm{N}$ & Median & Minimum & Maximum & p-value \\
\hline lung-Mean D & 8 & 482.9 & 27 & 1266 & 6 & 758.5 & 28 & 1548 & 0.414 \\
\hline lung-V1 & 8 & 40.2 & 8 & 97 & 6 & 88.8 & 72.0 & 96 & 0.029 \\
\hline lung-V5 & 8 & 25.7 & 11.3 & 48 & 6 & 42.5 & 27.6 & 74 & 0.020 \\
\hline lung-V20 & 8 & 18.3 & 2 & 32 & 6 & 30.8 & 19.7 & 37 & 0.020 \\
\hline lung-D50 & 8 & 107.5 & 39 & 674 & 6 & 107 & 12 & 1677 & 0.950 \\
\hline
\end{tabular}

Table 7. Comparison of different dosimetric parameters for both breasts in case of mediastinal and/or axillary irradiation.

\begin{tabular}{|c|c|c|c|c|c|c|c|c|c|}
\hline \multicolumn{10}{|c|}{ Radiotherapy Technique } \\
\hline & \multicolumn{4}{|c|}{ INRT } & \multicolumn{5}{|c|}{ IFRT } \\
\hline & $\mathrm{N}$ & Median & Minimum & Maximum & $\mathrm{N}$ & Median & Minimum & Maximum & $\mathrm{p}$-value \\
\hline Lt Breast-mean Dose & 7 & 129 & 33 & 482 & 4 & 264.5 & 223 & 353 & 0.315 \\
\hline Lt Breast-V1 & 7 & 12.1 & 1.5 & 33.4 & 4 & 32.3 & 28.4 & 33.4 & 0.042 \\
\hline Lt Breast-V5 & 7 & 6.7 & 0.7 & 16.9 & 4 & 15.3 & 3.2 & 17.8 & 0.315 \\
\hline Lt Breast-V20 & 6 & 1.8 & 0.3 & 9 & 4 & 3.5 & 0.4 & 5.6 & 0.476 \\
\hline Lt Breast-D50 & 7 & 12 & 2 & 57 & 4 & 85.9 & 82 & 96 & 0.006 \\
\hline Rt Breast-mean Dose & 7 & 88 & 28.8 & 426 & 4 & 56.9 & 27 & 245 & 0.412 \\
\hline Rt Breast-V1 & 7 & 4.8 & 0.3 & 23.7 & 4 & 32.5 & 27.6 & 36.4 & 0.006 \\
\hline Rt Breast-V5 & 6 & 8.7 & 0.3 & 16.4 & 4 & 15.2 & 2.5 & 18.3 & 0.257 \\
\hline Rt Breast-V20 & 6 & 2.1 & 0.2 & 12.3 & 4 & 3.7 & 0.3 & 4.9 & 0.914 \\
\hline Rt Breast-D50 & 7 & 19 & 2 & 48 & 4 & 77.5 & 61 & 83 & 0.006 \\
\hline
\end{tabular}

Table 8. Dosimetric data comparison for the heart and thyroid gland.

\begin{tabular}{|c|c|c|c|c|c|c|c|c|c|}
\hline \multicolumn{10}{|c|}{ Radiotherapy Technique } \\
\hline & \multicolumn{4}{|c|}{ INRT } & \multicolumn{4}{|c|}{ IFRT } & \multirow[b]{2}{*}{ p-value } \\
\hline & $\mathrm{N}$ & Median & Minimum & Maximum & $\mathrm{N}$ & Median & Minimum & Maximum & \\
\hline Heart-Mean Dose & 8 & 167 & 1.8 & 2263 & 6 & 260.5 & 89 & 1594 & 0.439 \\
\hline Heart-V20 & 7 & 23.1 & 1.4 & 42.8 & 6 & 36.9 & 9 & 94.9 & 0.116 \\
\hline Heart-V30 & 6 & 13.7 & 0.6 & 36.4 & 6 & 21.4 & 4 & 63.6 & 0.337 \\
\hline Thyroid-Mean Dose & 13 & 298 & 9 & 1284 & 17 & 197 & 3 & 3492 & 0.691 \\
\hline Thyroid-V1 & 13 & 46 & 1 & 99 & 17 & 1 & 1 & 98 & 0.017 \\
\hline Thyroid-V5 & 13 & 35 & 1 & 91.7 & 17 & 1 & 1 & 98.4 & 0.045 \\
\hline Thyroid-V20 & 13 & 22 & 1 & 98.6 & 17 & 36 & 1 & 98.9 & 0.833 \\
\hline Thyroid-D50 & 13 & 298 & 2 & 816 & 17 & 191 & 3 & 2986 & 0.933 \\
\hline
\end{tabular}


the treatment of Hodgkin's lymphoma and its impact on both progression free and overall survival many of the current clinical trials aim to either omit or minimize radiotherapy dose or volume with the intention to decrease the potential incidence of late radiotherapy toxicity including mainly carcinogenesis and cardio-vascular events.

The rationale for involved node radiation therapy is to further improve the therapeutic ratio, to reduce radiotherapy-induced morbidity in patients with limited-stage Hodgkin's lymphoma treated with chemo-radiotherapy while maintaining the excellent disease control achieved by conventional IFRT. This is based on the observation that after chemotherapy alone, most relapses of HL occur in the previously involved nodes; therefore, it has been extrapolated that the addition of INRT should be equivalent to IFRT in preventing local relapse while regional control will be accomplished by systemic chemotherapy [18].

Expert opinions in this regard can be sharply divided, especially in the approach to early stage, non-bulky disease and outcomes with radiotherapy omission are partly contingent upon the adequacy of the preceding chemotherapy $[19,20]$.

In the EORTC-GELA H9-F study for early favorable Hodgkin's lymphoma investigating reduction or omission of IFRT following complete remission to EBVP (epirubicin, bleomycin, vinblastine, and prednisone), no differences were seen between 20 and 36 Gy radiation on interim analysis [21]. However, the no-RT arm was closed due to excess relapse [21].

Wherever the role of RT is questioned, consultation with a radiation oncologist early in the treatment course is encouraged in order to have an informed discussion of risks versus benefits. Whether in limited or advanced Hodgkin's lymphoma, clear subsets of patients do benefit from the addition of radiotherapy. However, more accurate identification of such patients is needed. Whether interim 18F-FDG PET can help to distinguish these subsets of patients is the focus of ongoing trials [9,10].

Radiotherapy as a component of combined treatment modality was prospectively studied in a single clinical trial conducted at the British Colombia cancer center. In this trial 325 patients with limited-stage Hodgkin's lymphoma, diagnosed between May 1, 1989 and April 1, 2005, were treated with chemotherapy and radiation therapy following era-specific guidelines: Extended field radiotherapy (EFRT) until 1996; involved field radiotherapy (IFRT) from 1996 to 2001; involved nodal radiotherapy (INRT) from 2001 to 2005 . INRT defined as the pre-chemotherapy nodal volume with margins up to 5 $\mathrm{cm}$ to account for physiological movement, set-up variation, and the limitations of conventional simulation techniques [22]. The distribution of cases among the three radiation therapy groups were EFRT, 39\%; IFRT, 30\%; and INRT, $31 \%$. Median follow-up of living patients was 80 months. Median time to relapse was 37 months. Twelve relapses occurred: four after EFRT (3\%); five after IFRT (5\%); and three after INRT (3\%). No marginal recurrences occurred after INRT. At 5 years, progression-free survival (PFS) was 97\%, and overall survival (OS) was 95\% [22]. These results are similar to ours regarding relapse rate and disease free survival.

Regarding acute toxicity in our study no difference was found between the INRT and IFRT arms resulting from irradiation of upper airway, oral cavity or the pharynx. On the other hand, radiation pneumonitis incidence was higher in the IFRT arm for those receiving mediastinal irradiation. The reduction of included lung volume results in consequent reduction of lung toxicity in Hodgkin's lymphoma patients in the INRT group.

A study proposed by Amy M. et al. identified 75 patients with newly diagnosed HL treated with mediastinal RT (IFRT) and 17 patients with relapsed/refractory HL treated with mediastinal RT (IFRT) before or after transplant. Lung dose volumetric parameters including mean lung dose and percentage of lungs receiving 20 Gy were calculated. Radiation pneumonitis developed in 7 patients $(10 \%)$ who received mediastinal RT as a part of initial therapy. A mean lung dose of 13.5 Gy or greater was a predictor of radiation pneumonitis $(\mathrm{p}=0.04)$ and a $33.5 \%$ of lung volume receiving 20 Gy or greater significantly predicted for radiation pneumonitis $(p=0.009)$ [23].

Regarding Second malignancy and cardiac toxicity they are the most concerning of radiation-induced late morbidities, constituting the most common causes of non-lymphoma death in long-term Hodgkin's survivors [24].

Clinical studies had demonstrated that reducing the radiotherapy field size from EFRT to IFRT decreases the incidence of secondary malignancies. A meta-analysis of 10 randomized studies found that breast cancer risk was significantly higher in survivors of Hodgkin's lymphoma treated with EFRT compared with IFRT (odds ratio 3.25, $\mathrm{p}=0.04)$ [25].

Researchers predict that reducing supra-diaphragmatic radiotherapy fields from EFRT to IFRT reduces the excess relative risk of breast and lung cancers in females by $65 \%$ and lung cancers in males by $40 \%$ [26].

The use of the INRT concept in our study resulted in reduction of mean radiation volumes from $683 \mathrm{~cm}^{3}$ in IFRT to $217 \mathrm{~cm}^{3}$ and this reduction was statistically significant $(\mathrm{p}=0.009)$.

A trial Based on 20 computed tomography datasets of patients with early unfavorable mediastinal Hodgkin's using same definitions of IFRT and INRT found that 
PTV volume mean values were reduced from $1705 \mathrm{~cm}^{3}$ in IFRT cases to $1015 \mathrm{~cm}^{3}$ in INRT cases [27].

The reduction of radiation volume from IFRT to INRT across different trials and in this current study is associated with the added benefit of minimizing the radiation acute and late effects.

Moreover, the dose to risk organs in all published dosimetric analyses using INRT with conventional 3D techniques is associated with significant reduction of lung, breast, heart and thyroid gland radiation exposure which was noticed in both high dose and low dose regions particularly for the lungs and breasts. Interpretation of dosimetric plan comparison results may be limited by the use of different planning systems and different dose algorithms when comparing dosimetric results of different planning studies and this is particularly evident when comparing low dose exposure [27-29].

In our study the lung and breast doses in the case of mediastinal irradiation only cases showed a statistically significant difference between both groups. Lung doses including V1, V5, V20 were all significantly reduced in the INRT arm $(p=0.02)$, both breasts showed reduction in dosimetric parameters which were most obvious for V1 and D 50\%. Moreover, the dose reduction observed for the right breast was more compared to the left breast coinciding with other published data [27,29].

The reduction of heart doses in our study inspite of being numerically evident was not statistically significant in our series $(p=0.5)$.

Lastly, regarding the thyroid gland in our study there was evident reduction of the low dose values in the INRT $\operatorname{arm}(p=0.01)$ but not for the high dose regions $(p=0.8)$.

\section{Conclusions}

As yet there are no large datasets validating the use of involved nodal radiotherapy; these guidelines will emerge from the current generation of clinical trials.

Radiotherapy remains the most effective single modality in the treatment of Hodgkin's lymphoma. A reduction in both treatment volume and overall treatment dose should now be considered to minimize the risks of late sequelae. However, it is important that this is not at the expense of the excellent disease control currently achieved.

Close attention to radiotherapy technique is critical. This must include imaging protocols, effective immobilization, and outlining and treatment reproducibility. Verification is also essential.

\section{REFERENCES}

[1] A. Jemal, R. Siegel, E. Ward, et al., "Cancer Statistics," CA: A Cancer Journal for Clinicians, Vol. 56, No. 2,
2006, pp. 106-130. doi:10.3322/canjclin.56.2.106

[2] R. T. Hoppe, R. H. Advani, P. J. Bierman, et al., "Hodgkin Lymphoma: Clinical Practice Guidelines in Oncology," Journal of the National Comprehensive Cancer Network, Vol. 4, No. 3, 2006, pp. 210-230.

[3] G. Bonadonna, V. Bonfante, S. Viviani, et al., "ABVD plus Subtotal Nodal versus Involved-Field Radiotherapy in Early-Stage Hodgkin's Disease: Long-Term Results," Journal of Clinical Oncology, Vol. 22, No. 14, 2004, pp. 2835-2841. doi:10.1200/JCO.2004.12.170

[4] K. Ng, "Late Complications after Treatment for Hodgkin Lymphoma," Current Hematologic Malignancy Reports, Vol. 3, No. 3, 2008, pp. 119-125. doi:10.1007/s11899-008-0018-6

[5] K. Ng, M. P. Bernardo, E. Weller, et al., "Long-Term Survival and Competing Causes of Death in Patients with Early-Stage Hodgkin's Disease Treated at Age 50 or Younger," Journal of Clinical Oncology, Vol. 20, No. 8, 2002, pp. 2101-2108. doi:10.1200/JCO.2002.08.021

[6] P. Mauch, "Second Malignancies after Curative Radiation Therapy for Good Prognosis Cancers," International Journal of Radiation Oncology, Biology, Physics, Vol. 33, No. 4, 1995, pp. 959-960.

doi:10.1016/0360-3016(95)02141-9

[7] A. Engert, P. Schiller, A. Josting, et al., "Involved-Field Radiotherapy is Equally Effective and Less Toxic Compared with Extended-Field Radiotherapy after Four Cycles of Chemotherapy in Patients with Early-Stage Unfavourable Hodgkin's Lymphoma: Results of the HD8 Trial of the German HL Study Group," Journal of Clinical Oncology, Vol. 21, No. 19, 2003, pp. 3601-3608. doi:10.1200/JCO.2003.03.023

[8] V. B. Ad, O. Paltiel and E. Glatstein, "Radiotherapy for Early-Stage Hodgkin's Lymphoma: A 21st Century Perspective and Review of Multiple Randomized Clinical Trials," International Journal of Radiation Oncology, Biology, Physics, Vol. 72, No. 5, 2008, pp. 1472-1479. doi:10.1016/j.ijrobp.2008.08.026

[9] A. Gallamini, et al., "Early Interim PET Scan in Hodgkin Lymphoma: Where Do We Stand?" Leukemia \& Lymphoma, Vol. 49, No. 4, 2008, pp. 659-662. doi:10.1080/10428190801888704

[10] T. Kazama, S. C. Faria, V. Varavithya, S. Phongkitkarun, H. Ito, et al., "Macapinlac HA: FDG PET in the Evaluation of Treatment for Lymphoma: Clinical Usefulness and Pitfalls," Radiographics, Vol. 25, No. 1, 2005, pp. 191207. doi:10.1148/rg.251045045

[11] E. M. Noordijk, J. Thomas, C. Ferme, et al., "First Results of the EORTC-GELA Randomized Trials: The H9-F Trial (Comparing 3 Radiation Dose Levels) and the H9-U Trial (Comparing 3 Chemotherapy Schemes) in Patients with Favorable or Unfavorable Early Stage HL," Journal of Clinical Oncology, Vol. 23, No. 16S, 2005, p. 6505.

[12] J. M. P. Yahalom, "The Involved Feild is Back: Issues in Deleniating Thr Readiation Feild in HL," Annals of Oncology, Vol. 13, Suppl. 1, 2002, pp. 79-83.

[13] V. Diehl, C. Brillant, A. Engert, et al., "HD10: Investi- 
gating Reduction of Combined Modality Treatment Intensity in Early Stage Hodgkin's Lymphoma. Interim Analysis of a Randomized Trial of the German Hodgkin Study Group (GHSG)," Journal of Clinical Oncology, Vol. 23, No. 2005, pp. 16S-5561.

[14] T. Girinsky and M. Ghalibafian, "Radiotherapy of Hodgkin Lymphoma: Indications, New Fields, and Techniques," Seminars in Radiation Oncology, Vol. 17, No. 3, 2007, pp. 206-222. doi:10.1016/j.semradonc.2007.02.007

[15] H. T. Eich, et al., "Involved-Node Radiotherapy in EarlyStage Hodgkin's Lymphoma. Definition and Guidelines of the German Hodgkin Study Group (GHSG)," Strahlentherapie und Onkologie, Vol. 184, No. 8, 2008, pp. 406-410. doi:10.1007/s00066-008-1882-7

[16] L. G. R. Specht, M. J. Clarke, et al., "Influence of More Extensive Radiotherapy and Adjuvant Chemotherapy on Long-Term Outcome of Early-Stage Hodgkin's Disease: A Meta-Analysis of 23 Randomized Trials Involving 3888 Patients," Journal of Clinical Oncology, Vol. 16, No. 3, 1998, pp. 830-843.

[17] O. W. Press, M. LeBlanc, A. S. Lichter, et al., "Phase III Randomized Intergroup Trial of Subtotal Lymphoid Irradiation Versus Doxorubicin, Vinblastine, and Subtotal Lymphoid Irradiation for Stage IA to IIA Hodgkin's Disease," Journal of Clinical Oncology, Vol. 19, No. 22, 2001, pp. 4238-4244.

[18] M. Shahidi, et al., "Site of Relapse after Chemotherapy Alone for Stage I and II Hodgkin's Disease," Radiotherapy \& Oncology, Vol. 78, No. 1, 2006, pp. 1-5. doi:10.1016/j.radonc.2005.10.018

[19] J. Yahalom, "Don't Throw out the Baby with the Bathwater: On Optimizing Cure and Reducing Toxicity in Hodgkin's Lymphoma," Journal of Clinical Oncology, Vol. 24, No. 4, 2006, pp. 544-548. doi:10.1200/JCO.2005.04.4396

[20] G. P. Canellos, "Chemotherapy Alone for Early Hodgkin's Lymphoma: An Emerging Option," Journal of Clinical Oncology, Vol. 23, No. 21, 2005, pp. 4574-4576. doi:10.1200/JCO.2005.01.911

[21] H. Eghbali, P. Brice, G. Creemers, et al., "Comparison of Three Radiation Dose Levels after EBVP Regimen in Favorable Supra-Diaphragmatic Clinical Stages (CS) I-II Hodgkin's Lymphoma (HL): Preliminary Results of the EORTC-GELA H9-F Trial," Blood, Vol. 106, No. 11, 2005, p. 814.

[22] B. A. Campbell, N. Voss, T. Pickles, et al., "Involved-
Nodal Radiation Therapy as a Component of Combination Therapy for Limited-Stage Hodgkin's Lymphoma: A Question of Field Size," Journal of Clinical Oncology, Vol. 26, No. 32, 2008, pp. 5170-5174. doi:10.1200/JCO.2007.15.1001

[23] A. M. Fox, A. P. Dosoretz, P. M. Mauch, Y. H. Chen, D. C. Fisher, A. S. LaCasce, A. S. Freedman, B. Silver and A. K. Ng, "Predictive Factors for Radiation Pneumonitis in Hodgkin Lymphoma Patients Receiving CombinedModality Therapy," International Journal of Radiation Oncology, Biology, Physics, Vol. 83, No. 1, 2012, pp. 277-283.

[24] B. M. Aleman, A. W. van den Belt-Dusebout, W. J. Klokman, et al., "Long-Term Cause Specific Mortality of Patients Treated for Hodgkin's Disease," Journal of Clinical Oncology, Vol. 21, No. 18, 2003, pp. 3431-3439. doi:10.1200/JCO.2003.07.131

[25] J. Franklin, A. Pluetschow, M. Paus, et al., "Second Malignancy Risk Associated with Treatment of Hodgkin's Lymphoma: Meta-Analysis of the Randomised Trials," Annals of Oncology, Vol. 17, No. 12, 2006, pp. 17491760. doi:10.1093/annonc/mdl302

[26] E.-S. Koh, T. H. Tran, M. Heydarian, et al., "A Comparison of Mantle versus Involved Field Radiotherapy for Hodgkin's Lymphoma: Reduction in Normal Tissue Dose and Second Cancer Risk," Radiation Oncology, Vol. 2, No. 3, 2007, p. 13. doi:10.1186/1748-717X-2-13

[27] J. Koeck, Y. Abo-Madyan, F. Lohr, F. Stieler, J. Kriz, R. P. Mueller, F. Wenz and H. T. Eich, "Radiotherapy for Early Mediastinal Hodgkin Lymphoma According to the German Hodgkin Study Group (GHSG): The Roles of Intensity-Modulated Radiotherapy and Involved-Node Radiotherapy," International Journal of Radiation Oncology, Biology, Physics, Vol. 83, No. 1, 2012, pp. 268276.

[28] D. C. Weber, et al., "Involved-Node and Involved-Field Volumetric Modulated Arc vs. Fixed Beam IntensityModulated Radiotherapy for Female Patients with EarlyStage Supra-Diaphragmatic Hodgkin Lymphoma: A Comparative Planning Study," International Journal of Radiation Oncology, Biology, Physics, Vol. 75, No. 5, 2009. pp. 1578-1586. doi:10.1016/j.ijrobp.2009.05.012

[29] B. A. Campbell, et al., "Minimising Critical Organ Irradiation in Limited Stage Hodgkin Lymphoma: A Dosimetric Study of the Benefit of Involved Node Radiotherapy," Annals of Oncology, Vol. 23, No. 5, 2012, pp. 1259-1266. doi:10.1093/annonc/mdr439 